\title{
La sclérothérapie des varices œesophagiennes n'est pas efficace dans la prophylaxie primaire des hémorragies digestives chez le cirrhotique
}

La sclérothérapie endoscopique des varices œesophagiennes est largement utilisée dans la prévention des récidives d'hémorragies digestives par hypertension portale chez le cirrhotique. Elle prévient ces récidives de façon efficace et plusieurs études contrôlées indiquent que ce traitement pourrait améliorer la survie. La question se pose donc de savoir si elle est également utile dans la prévention de la première hémorragie (prophylaxie primaire). Quatre études contrôlées ont montré que dans ce cas, la sclérothérapie pourrait également être utile pour prévenir les hémorragies, mais ces études ont été contestées, notamment parce que la fréquence des hémorragies dans les groupes témoins était anormalement haute. Une cinquième étude n'a montré aucun bénéfice sur les hémorragies et son effet global est plutôt délétère en raison des complications propres à la technique.

L'équipe gastroentérologique de Munich vient de publier les résultats d'une sixième étude contrôlée portant sur 133 patients [1]. Tous avaient une cirrhose, alcoolique dans $66 \%$ des cas. Les critères de sélection ont été les suivants : (1) existence d'une cirrhose ; (2) au moins deux varices du tiers inférieur de l'œsophage, d'un diamètre d'au moins $5 \mathrm{~mm}$; (3) pas d'antécédent d'hémorragie digestive; (4) pas de maladie extrahépatique menaçant la vie du malade à court terme; (5) pas d'ulcère gastro-duodénal ; $(6)$ un score de gravité de Child-Pugh de moins de 12 (ce qui exclut les malades ayant une insuffisance hépatique très sévère); (7) pas de traitement par $\beta$ bloquants, pénicillamine ou corticostéroïdes ; (8) âge de 18 à 75 ans. Tous les malades ont été informés des conditions de l'essai.

Après tirage au sort, les malades ont été répartis en deux groupes: un rothérapie, et un groupe témoin de 65 malades ne recevant aucun traitement. Les auteurs se sont assurés de façon très soigneuse que les deux groupes avaient au départ des caractéristiques identiques quant au degré d'insuffisance hépatique, aux constatations endoscopiques ou à la cause de la cirrhose. Tous les malades ayant eu une hémorragie digestive ont été, ultérieurement, traités par sclérose des varices quand cela a été possible.

Au cours d'un suivi moyen de 22 mois, une hémorragie par rupture de varices est survenue chez $28 \%$ des malades traités par sclérothérapie et $37 \%$ des témoins, une différence non significative. Le taux de mortalité a été de $35 \%$ dans le groupe traité et de $46 \%$ dans le groupe témoin, et les courbes actuarielles de survie ont été similaires. (De façon un peu surprenante cependant, les auteurs signalent une survie un peu meilleure chez les patients ayant une cirrhose alcoolique de sévérité modérée malgré une incidence identique d'hémorrragies digestives).

Cette étude montre donc que la sclérothérapie n'est pas efficace dans la prophylaxie primaire des hémorragies digestives chez le cirrhotique (ou, tout au moins, que s'il y a un bénéfice, celui-ci est probablement très faible: l'incidence des hémorragies $a$, en effet, été légèrement inférieure dans le groupe traité à celle du groupe témoin, mais il aurait fallu 446 malades pour affirmer que cette différence était ou non significative). Ajoutée aux autres études déjà publiées sur le sujet, elle renforce très largement l'impression que la sclérothérapie n'est pas indiquée pour la prophylaxie primaire des hémorragies digestives chez le cirrhotique. Il n'est cependant pas tout à fait exclu que certains sous-groupes puissent tirer bénéfice de la méthode: les auteurs insistent sur la meilleure sur- vie chez les cirrhotiques alcooliques de sévérité moyenne. Il est clair que les études à venir devront porter en priorité sur les malades à très haut risque hémorragique, c'est-à-dire ceux ayant des grosses varices œesophagiennes, des signes « rouges » à l'endoscopie (c'est-à-dire des lésions muqueuses œesophagiennes sur la paroi des varices) et ceux ayant à la fois des varices œesophagiennes et des varices de la grosse tubérosité gastrique. A l'heure actuelle, la seule mesure ayant montré une certaine efficacité en prophylaxie primaire est le traitement par les $\beta$-bloquants.

S. E.

1. Sauerbruch T, Wotzka R, Köpcke W, et al. Prophylactic sclerotherapy before the first episode of variceal hemorrhage in patients with cirrhosis. N Engl J Med 1988; 319 : 815.

\section{BRÈVES}

Exclusion allélique et exclusion isotypique. Des souris transgéniques exprimant un gène $\beta$ réarrangé (codant pour la chaîne $\beta$ du récepteur $\alpha \beta$ des lymphocytes $T$ ) ont été créées [1]. Leurs cellules $T$ ne réarrangent pratiquement pas les gènes $\beta$ et $\gamma$ endogènes. Le gène $\gamma$ code pour la chaîne $\gamma$ du second récepteur pour l'antigène connu, de formule « $\gamma \delta »$. Ainsi, l'expression d'un gène réarrangé inhibe-t-elle le réarrangement ultérieur d'un gène allélique $(\beta)$ et d'un gène non allélique $(\gamma)$. Ce dernier phénomène peut être appelé « exclusion isotypique». Il empêche une cellule $T$ d'avoir une double spécificité d'antigène (idiotypique) secondaire à l'expression des deux types de récepteur, $\alpha \beta$ et $\gamma \delta$.

[1. Fenton RG, et al. Science 1988; 241 : 1089-92.] 